\title{
Detection and Localization of Gd-DTPA Following Dynamic Contrast Enhanced Magnetic Resonance Imaging (dMRI) Using Cryo Field Emission Scanning Electron Microscopy (FESEM) and Energy Dispersive X-ray Spectrometry (EDS)
}

\author{
C.A. Ackerley ${ }^{*}$, M.D. Noseworthy ${ }^{* *}$, A. Tilups ${ }^{*}$, G.A. Wright ${ }^{* * *}$, and L.E. Becker ${ }^{*}$ \\ *Department Paediatric Laboratory Medicine, Hospital for Sick Children, Toronto, Canada \\ *** Department of Diagnostic Imaging, Hospital for Sick Children, Toronto, Canada \\ ${ }^{* * * *}$ Sunnybrook \& Women's College Health Sciences Centre, Toronto, Canada
}

A non-invasive method to evaluate microcirculation is dynamic magnetic resonance imaging (dMRI) (1). This technique involves the rapid acquisition of images while an intravenously injected contrast agent circulates. The image signal intensity is changed in a way that reflects the micro vascular environment. It is possible to obtain estimates of tissue blood volume, blood flow, and capillary permeability. This method assumes that contrast agents such as gadolinium diethylnetriaminepentaacetic acid (Gd-DTPA) remain intravascular and diffuse only into the perivascular spaces surrounding "leaky vessels". Using this approach tumour characterization can be done in soft tissues more readily than using CT or other radiological approaches. In this study we used cryo-analytical FESEM of both muscle and tumour vascular tissue in an animal model to verify the subcellular location of Gd-DTPA following enhanced dMRI scans.

New Zealand white rabbits (4-5kg) were implanted with $\sim 0.7 \times 10^{6}$ VX2 tumour cells, intramuscularly (biceps femoris). Dynamic MRI was performed 21 days following implantation. Anaesthetized animals were imaged using a $1.5 \mathrm{~T}$ system and then a $\mathrm{T}_{1} \mathrm{map}$, modified for spiral $\mathrm{k}$ space acquisition was acquired prior to contrast agent administration (2). Dynamic MRI was performed using a T1-weighted SPGR sequence to track an IV bolus injection of Gd-DTPA. Data was modeled according to Tofts et al (3). Pixel-wise analysis produced parametric maps of enhancement slope, time to peak, capillary permeability and perivascular volume. Following MR scanning, $1 \mathrm{~mm}^{3}$ portions of muscle and tumour were removed and immediately fixed by plunge freezing the tissues into either liquid pentane or ethane. In addition whole blood was collected from the animals and prepared in the same fashion. Frozen samples were then cryo-fractured in a cryopreparative chamber attached to a FESEM and rendered conductive by sputter coating them with a thin layer of chromium. Specimens were then transferred to a cold stage and examined in the microscope at temperatures lower than $-155^{\circ} \mathrm{C}$. Analyses were performed under standardized conditions using EDS. At least 25 areas were analyzed from each of the following areas; the lumina of the blood vessels, the endothelial cells and in the perivascular spaces. Both muscle and tumour samples as well as the frozen whole blood sample were analyzed in this manner. The frozen whole blood was used as a qualitative standard. Remaining tissues were fixed in either formalin or glutaraldehyde and processed for either routine light or transmission electron microscopic examination.

From dMRI signal modeling results the tumour tissue was judged as hypervascular, relative to the surrounding muscle. Gd-DTPA markedly increased MR image signal in the tumour in comparison 
to the surrounding normal muscle. Histological and ultrastructural examination of routinely processed tumour samples revealed a highly vascularized tumour consisting primarily of polygonal shaped cells growing between the muscle fibers where the tumour cells had been seeded.

Between 10 and $20 \mu \mathrm{m}$ of acceptable freezing with good ultrastructure was achieved using plunge freezing. Results were more consistent when ethane was used as the cryogen. Gd was detected in the lumina of the blood vessels in both muscle and tumour tissue at concentrations comparable to the whole blood samples. Perivascular gadolinium was detected only in the tumour tissue, due to the permeable nature of these blood vessels. Ultrastructural examination of these vessels showed a thinner endothelial cell and almost no intact basal lamina. Perivascular Gd concentration was at least 4 fold of that found in the whole blood samples and in the lumina of the blood vessels. Perivascular $\mathrm{Gd}$ was not detected in the muscle tissue. Contrary to current concepts, Gd was detected in the endothelial cells of both the tumour and muscle blood vessels. The mathematical modeling on which dMRI is based does not take into account the presence of an endothelial cell pool of Gd. As a result, an overestimation of both blood and perivascular volume using this technique is possible. Further studies into the quantity and the efficacy of potential transport mechanisms of Gd through the endothelial cells are required in order to fully understand this phenomena and are the basis of ongoing work in our laboratory.

\section{References}

1. P.S. Tofts, J Magn Reson Imag, 7 (1997) 91.

2. M.D. Noseworthy et al., J Magn Reson Imag, 9 (1999) 814.

3. P.S. Tofts et al., Magn Reson Med, 33 (1995) 564.
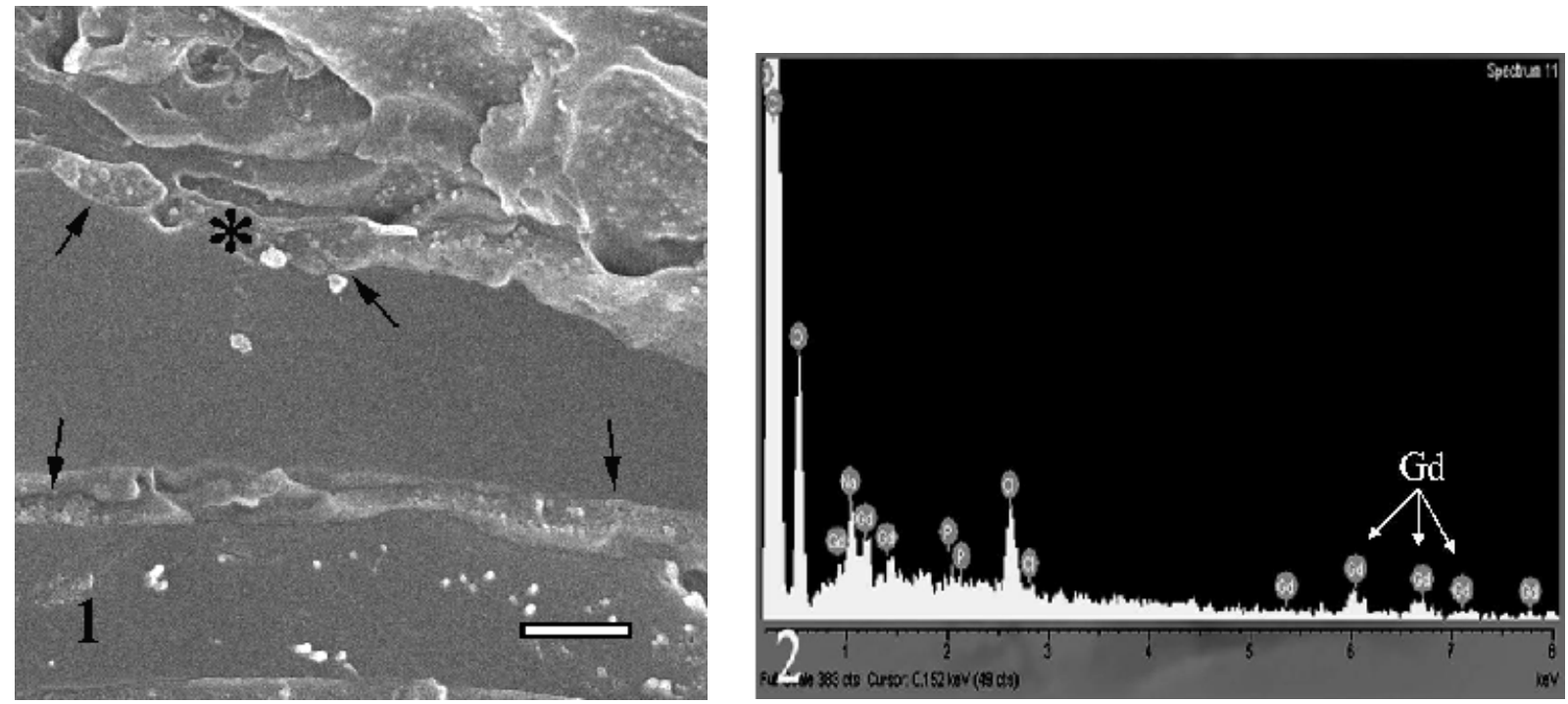

Fig.1. Cryo FESEM image of a fracture through a tumour blood vessel. Note the numerous vesicles evident in the cytoplasm of the endothelial cell (arrows). Asterisk indicates area analysed by EDS. Bar equals $1 \mu \mathrm{m}$.

Fig.2. EDS spectra from the area marked by the asterisk in figure 1. 